\title{
Transient simulation of wear in a lobe pump using the wear processor
}

\author{
Y. J. Chen ${ }^{1,2}$ \& N. Huber ${ }^{2,3}$ \\ ${ }^{I}$ Institut für Zuverlässigkeit von Bauteilen und Systemen, \\ Universität Karlsruhe(TH), Germany \\ ${ }^{2}$ Institut für Werkstoffphysik und Technologie, \\ Technische Universität Hamburg-Harburg, Germany \\ ${ }^{3}$ Institut für Werkstoffforschung, \\ GKSS-Forschungszentrum Geesthacht GmbH, Germany
}

\begin{abstract}
Lobe pumps represent an intelligent design principle for fluid technology. They combine competence in design and complex high-precision manufacturing processes and find their application in the fields of automotive and mobile hydraulics industry. The chambers for inlet, movement and outlet of the fluid are formed in a continuous process by rotating gear-shaped parts, which are sealed by several sliding contacts. The ability to predict wear in such complex systems is essential for the development and lifetime prediction. Using a dynamic 2D finite element simulation, the transient contact pressure and slip conditions are obtained on the surfaces of the three parts of which the pump consists: inner rotor, outer rotor and housing. For the wear simulation it is necessary to handle simultaneous wear in multiple contacts and contacting surfaces. It is shown that it is possible to predict wear in such a system over a large number of rotations with considerable effect on the geometry of the different components. The simulations allow one to predict the number of rotations for a given wear coefficient until critical gaps are formed, which can significantly reduce the performance of the lobe pump. Furthermore, the wear profiles show that the wear between the outer rotor surface and the housing reflects the pressure difference in the pump as well as the symmetry of the rotors. This work shows that it is possible to predict wear and the changing kinematics of systems, which are based on moving contacts. Problems of future interest are gears, camshaft follower systems, or injection systems.
\end{abstract}

Keywords: wear, wear prediction, transient contact, numerical simulation. 


\section{Introduction}

Lobe pumps represent an intelligent design principle for fluid technology. They combine competences in design and complex high-precision manufacturing processes and find their application in the fields of automotive and mobile hydraulics industry. The pump design investigated in this work uses lobe rotors that turn continuously and has been fabricated in miniaturized dimensions [1]. An investigation with respect to short term fatigue and fracture of the lobe pumps has been carried out considering dynamic effects, friction, fabrication tolerances as well as braking and damping of fluid [2]. The time dependent forces and stress distributions in the components were found to be not critical with regard to failure due to short term fatigue or fracture. However, preliminary wear predictions under dry wear conditions indicated that the life time of the lobe pump might be short partially because severe wear occurs in the internal parts of the lobe pump $[1,3]$. However, these results should be considered as a rough guess due to the complex transient behavior of multiple sliding contacts. Based on authors' best knowledge, there is still no literature available in dealing with the wear response of such systems and a realistic prediction of wear is not possible so far.

For wear simulation, Archard's wear model is the most popular model as discussed by many authors [4-14]. Archard's wear law represents a linear relationship between the incremental wear, local contact pressure and sliding distance. It was originally formulated on the global component scale in the form

$$
\frac{V_{w}}{s}=k_{D} F_{N},
$$

where $V_{\mathrm{w}}$ is the wear volume, $k_{\mathrm{D}}$ is the dimensional wear coefficient, $s$ is sliding distance and $F_{\mathrm{N}}$ is the applied normal force. To determine the local wear distribution in a tribosystem, commonly the local formulation is used,

$$
\frac{h}{s}=k_{D} p \text {, }
$$

where $p$ is the contact pressure at a point on the surface. For a further generalization towards sliding distance dependent or time dependent wear prediction, eqn (2) needs to be formulated a differential equation [6]

$$
\frac{d h}{d s}=k_{D} p,
$$

with which an initial value problem can be solved through integration of the contact pressure over the slip distance. Therefore, the wear for each surface point results from the solution of the integral

$$
h_{w}=k_{D} \int p d s=k_{D} \int p(t) \frac{d s(t)}{d t} d t .
$$

In recent years, numerical simulation has achieved increasing attention as a powerful way for wear prediction and component design. As soon as multiple 
contacts and dynamic effects come into play, Finite Element simulations are so far the only way to achieve results, which account for the local contact conditions in the context of the system behavior. For well defined contact situations, for example, in gears, the global and local behavior has been studied with different multi-scale approaches $[6,11,13]$.

In the case of the micro lobe pump, contacts show a wide variation in their extension from conformal to Hertzian type. Also the number of contacts in the system changes by opening and closing of contacts as a function of the rotation angle. These circumstances don't allow an application of efficient models, such as Winkler surfaces. Thus, the combination of a finite element simulation with wear prediction using the Wear-Processor are applied here and details are given in the next section. The wear predictions for a single wear step and for multiple wear steps as well as the gap and changing of the kinematics are presented in the third part, which is followed by the conclusions.

\section{Finite element model}

The finite element code ABAQUS [15] was used for the analysis of the micro lobe pump. The simulation with multiple sliding contacts was solved by the ABAQUS-Explicit, which has proven excellent in dealing with transient and nonlinear effects as well as finite rotations.

\subsection{Geometry}

The finite element models used in this paper are based on those used in [2]. For the completeness of this paper, the basic information of the finite element model is stated here.

The finite element model as shown in Figure 1 consists of four different parts: the housing, outer rotor, inner rotor and inner shaft. The outer diameter of the housing is $2.0 \mathrm{~mm}$. These different parts are plugged together and, in this way, the different clearances can be taken into consideration. We assume zero initial clearance between the inner rotor and the inner shaft, while the clearances between the housing and the outer rotor and between the outer and inner rotor are non-zero, respectively (see Table 1). To study the influence of different clearances between the inner and outer rotor on the wear response, two different finite element meshes were adopted in this study.

Table 1: $\quad$ Contact definitions and variation of clearance.

\begin{tabular}{cccc}
\hline Contact no. & Outerpart & Inner part & Clearance $[\mu \mathrm{m}]$ \\
\hline 1 & Housing & Outer rotor & 1 \\
2 & Outer rotor & Inner rotor & 0,1 \\
3 & Inner rotor & Inner shaft & 0 \\
\hline
\end{tabular}

The outer edge of the housing is fixed in all directions. The inner shaft is turned by a rigid surface, which is adjusted and tied to the inner circular edge of the inner shaft and which is connected to a reference node with only the rotational degree of freedom available. The simulation of this micro pump model 


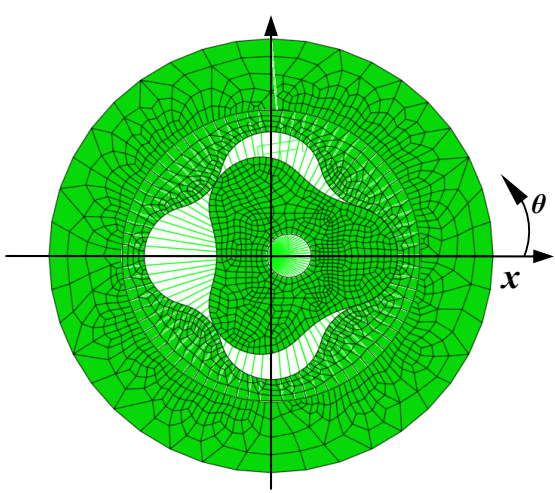

Figure 1: The finite element model of the micro pump.

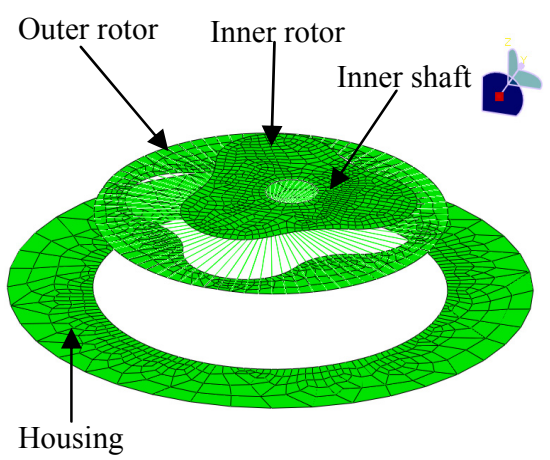

Figure 2: Detail configuration of the finite element model of the micro pump.

includes two steps. The first one is the acceleration step. The final angular velocity of 20,000 rpm at the inner shaft is applied with a constant acceleration when the inner shaft rotates to an angle of $120^{\circ}$. In the second step, the inner rotor rotates with the constant angular velocity of $20,000 \mathrm{rpm}$, and the simulation in this step is continued for $12 \mathrm{~ms}$ so that the inner rotor will rotate for 4 turns. The 4 turns ensure that the wear is the same with respect to the symmetry of the inner and outer rotors due to their 3 and 4 fold symmetry, respectively. The torque and force applied to this finite element model are the same as those in [2].

\subsection{Material properties}

In the finite element models, the housing and the rotors are made of ceramic while the inner shaft is made of steel. The behavior of the two materials is assumed to be elastic with the corresponding material constants given in Error! Reference source not found.. A Coulomb friction law with a friction coefficient $\mu=0.15$ is applied to all contact surfaces. The wear coefficient has been chosen with $k_{\mathrm{D}}=13.5 \times 10^{-8} \mathrm{~mm}^{3} / \mathrm{Nmm}[13,14]$.

Table 2: Material parameters.

\begin{tabular}{cccc}
\hline Material & $\rho\left[\mathrm{g} / \mathrm{cm}^{3}\right]$ & $\mathrm{E}[\mathrm{GPa}]$ & $v[-]$ \\
\hline $\mathrm{Al}_{2} \mathrm{O}_{3}$ & 3.99 & 400 & 0.23 \\
$\mathrm{Steel}$ & 7.80 & 210 & 0.3 \\
\hline
\end{tabular}

\subsection{Wear processor implementation}

Based on the previous Wear-Processor 5.2 which has been described in detail in $[8,16]$, several changes have been implemented. Some are visible from the flow chart in Figure 3, the most important, relevant for this work, are explained below. 


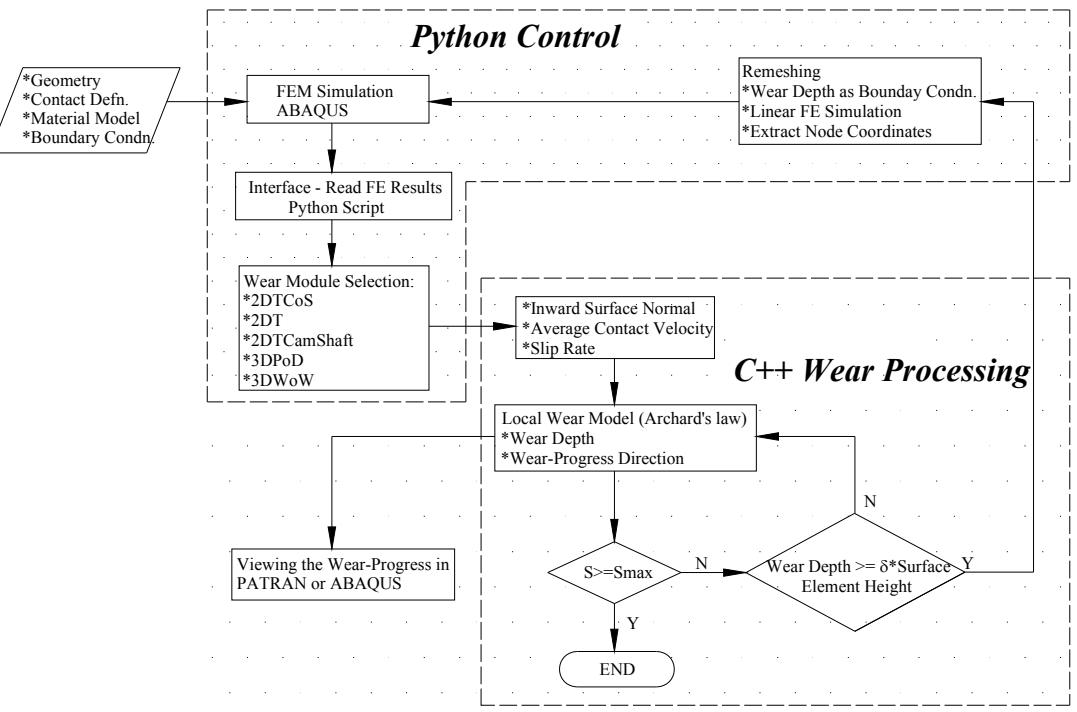

Figure 3: $\quad$ Flow chart for the working of Wear-Processor 6.0.

\section{A) Contact variables}

The latest version of ABAQUS 6.8.1 [15] provides the contact pressure $p$ of the slave and master directly. Besides, in the ABAQUS-Explicit solver, the slip variables are provided for both the slave surface and master surface, thus, the mapping procedure of the slip rate from the slave surface to that of the master surface $[8,16]$ will not needed in the present case.

B) Python control

The flow chart can now be separated into two parts; the python control part and the $\mathrm{C}++$ wear processing part. Preparation and starting of the contact simulation, reading FE results interface, wear module calling, and re-meshing process are integrated in the python control part. The $\mathrm{C}++$ wear processing part of the code will carry out the wear calculation for each corresponding wear module. Applications with the respective modules have been published earlier for a 3D pin-on-disc [8, 16], a twin wheel tribometer [9] and a 2D gear tooth [13].

\section{Results and discussions}

\subsection{Single wear step}

The wear simulations are carried out for initial clearances between the outer and inner rotor of 0 and $1 \mu \mathrm{m}$ (see Table 1 ), respectively. In the contact simulation, the inner rotor is rotated for 4 turns, and thus the outer rotor will rotate for 3 turns because the transmission ratio between the inner rotor and the outer rotor is 3:4. Actually, the wear of the components of the micro pump is very small after 
one contact simulation, and it will not have a large effect for the following contact simulation since the geometry change is quite insignificant. Thus, in one wear step, we can calculate a certain amount of wear based on one contact simulation. In this work, the number of wear increments in one wear step is 50 . Thus, one wear step assumes 200 rotations of the inner rotor.

The wear distribution of the inner rotor, outer rotor and the housing with and without initial clearance are shown in Figures 4-7.
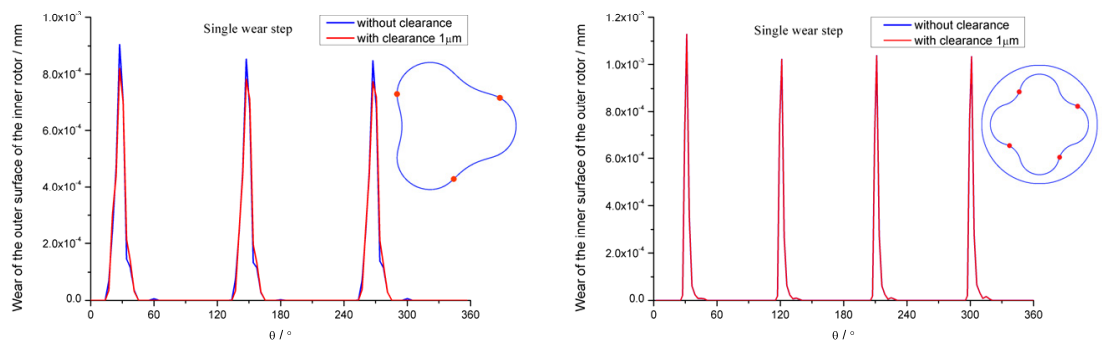

Figure 4: Wear of the outer
surface of the inner rotor.

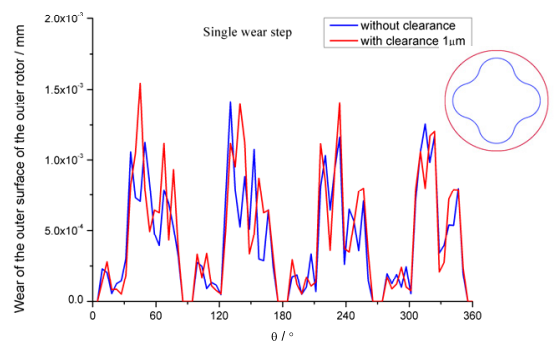

Figure 6: Wear of the outer rotor.

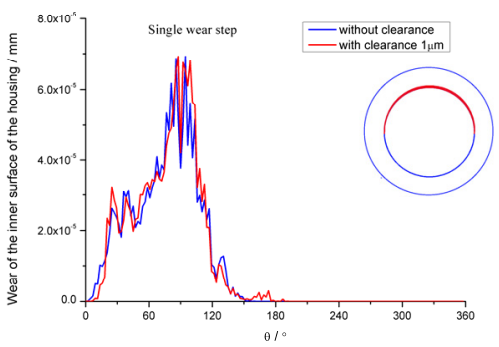

Figure 5: $\begin{aligned} & \text { Wear of the inner } \\ & \text { surface of the outer } \\ & \text { rotor }\end{aligned}$
rotor.

Figure 7: Wear of the inner surface of the housing.

Due to the geometric symmetry, a periodic behavior of the wear can be observed in the outer surface of the inner rotor as well as in the inner and outer surface of the outer rotor. The variable $\theta$ in the abscissa of these figures denotes the angle of each surface node with respect to the $x$ axis in the counter-clockwise direction, as shown in Figure 1. The maximum wear of the outer surface of the inner rotor occurs at $27^{\circ}, 147^{\circ}$ and $267^{\circ}$, while the maximum wear of the inner surface of the outer rotor occurs at $30^{\circ}, 120^{\circ}, 210^{\circ}$ and $300^{\circ}$. 


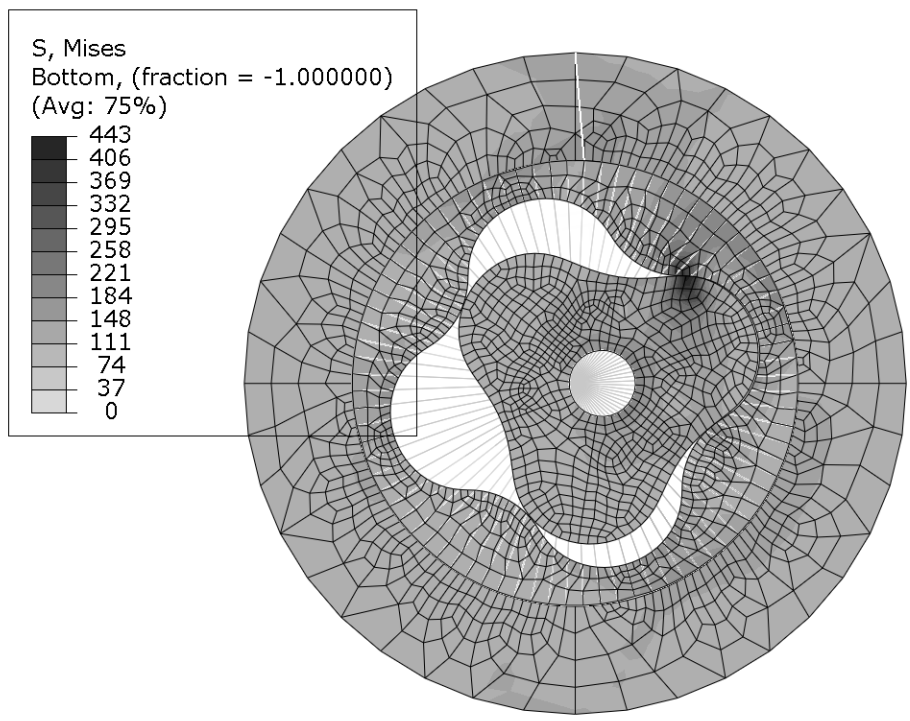

Figure 8: $\quad$ Von Mises stress distribution for turning angle $\theta=30$.

These non-zero wear points are marked in the corresponding figure. Due to the upward applied force resulting from the pressure difference of the lobe pump, the maximum contact pressure always appears at the upper right contact region of the inner rotor and outer rotor contact pair, as shown in Figure 8. For the inner surface of the housing, only the top half of the surface experiences contact due to the upward force applied at the geometrical center of the outer rotor, and hence wear occurs only at this top region.

It can be seen from Figures 4-7 that the initial clearance between the inner rotor and outer rotor has little influence on the wear of the rotors and the housing. The wear in these figures is almost comparable in the respective whole surface.

\subsection{Multiple wear steps}

In this section, the simulation is carried out with the same parameters as those in section 3.1. The progress of wear of the inner rotor, outer rotor and the housing are shown in Figures 9-12.

As shown in Figures 9-11, the wear of the inner rotor and outer rotor approaches to a very stable periodic shape with the increasing of wear steps. The maximum amount of wear is exactly the same for the 3 teeth of the inner rotor, and also for the 4 teeth of the outer rotor. The worn areas of the outer surface of the inner rotor as well as the inner surface of the outer rotor increase with increasing number of wear steps. Furthermore, from these results it can be concluded that the initial clearance does have only little effect on the final wear distribution of the micro pump. 


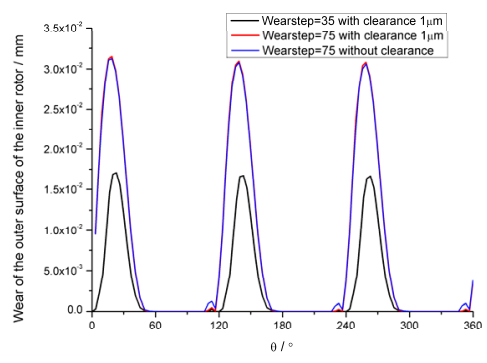

Figure 9: Wear of the outer surface of the inner rotor after multiple wear steps.

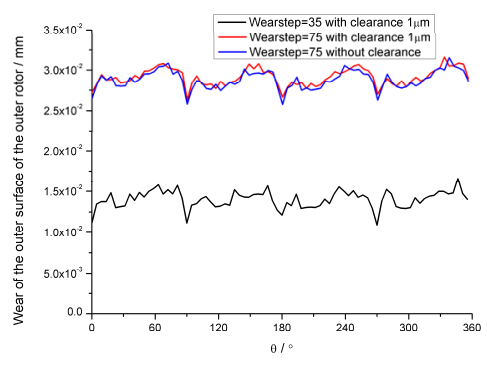

Figure 11: Wear of outer surface of the outer rotor after multiple wear steps.

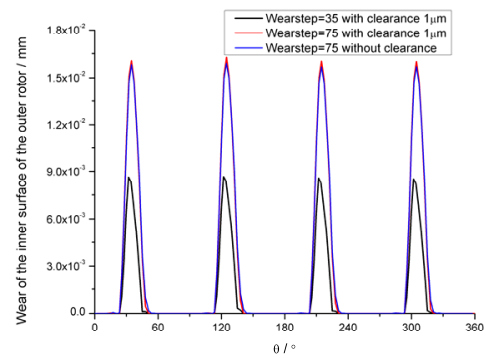

Figure 10: Wear of the inner surface of the outer rotor after multiple wear steps.

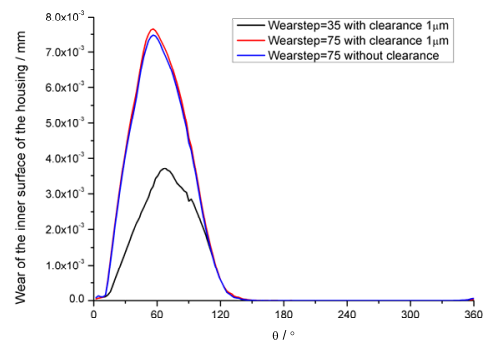

Figure 12: Wear of inner surface of the housing after multiple wear steps.

The wear distribution of the inner rotor, outer rotor and the housing show several interesting characteristics. After 75 wear steps, the maximum wear on the outer surface of the inner rotor is almost twice of that of the inner surface of the outer rotor. However, as we can see from Figures 4 and 5 for the first wear step, the maximum of wear of the outer surface of the inner rotor is smaller than that of the inner surface of the outer rotor. Thus, the rate of wear progress is not linear for the different contact surfaces. This indicates that a multiple wear step simulation is essential for the prediction of wear in such a complex dynamic system.

Furthermore, it can be seen from Figure 9 that the position of the maximum wear shifts left with increasing number of wear steps while the position of the maximum wear in shifts right. Also, the position of maximum wear of the inner surface of the housing in shifts left from $\theta=90^{\circ}$ for the first wear step to $56^{\circ}$ for the $75^{\text {th }}$ wear step. These phenomena can be explained by the relative motions 
between the inner rotor, outer rotor and the housing. The outer surface of the housing is fixed in space, and the inner rotor which is controlled by a reference point can only rotate around with its geometric center. However, the outer rotor doesn't have a fixed rotation center, and therefore it can move dependent on the forming gaps due to wear. As a result, the center of the outer rotor gradually approaches to the upper right corner. As a consequence, the position of maximum contact pressure on the outer surface of the inner rotor moves to a smaller angle $\theta$. For the position of maximum contact pressure on the inner surface of the housing we observe the same behavior. While for the outer rotor, it can be found that the position of the maximum contact pressure approaches a larger angle. Finally the change of the region with maximum contact pressure leads to the observed movement of the position of the maximum wear.

\subsection{Gap formation and change of kinematics}

The gaps between the inner rotor and the outer rotor (cf. Figures 8 and 16) as well as between the outer rotor and the housing increase with increasing number of wear steps, as shown in Figure 13. It can be seen that the gaps show a nearly linear increase. Using such a diagram, the maximum allowed number of rotations of the micro-pump can be predicted for a given maximum permitted gap.

Figure 14 shows the movement of the center of the outer rotor with increasing wear. Due to the upward force applied at the center of the outer rotor, the ycoordinate is always positive. In the very beginning, the outer rotor can rotate uniformly in the chamber and thus the x-movement in the horizontal direction is symmetric around the origin.

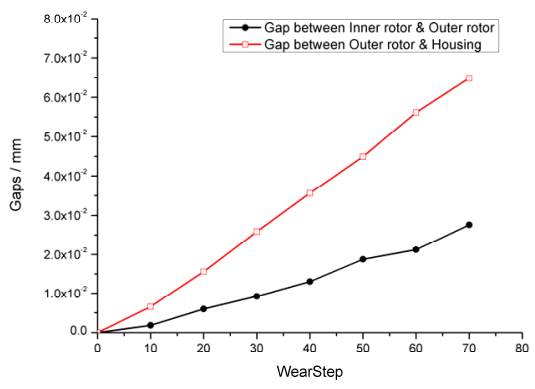

Figure 13: Gap formation with increasing number of wear steps.

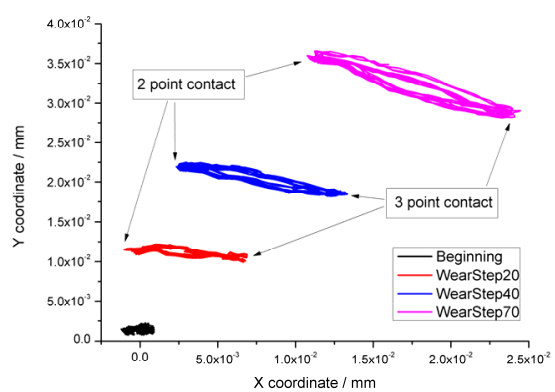

Figure 14: The movement of the center of the outer rotor with increasing wear step. 
58 Tribology and Design

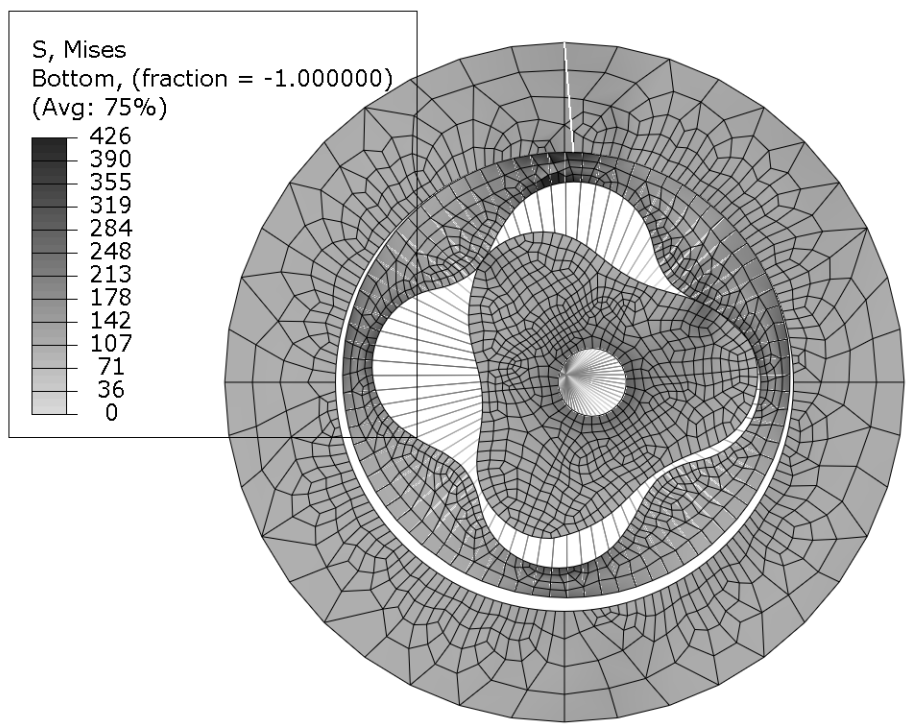

Figure 15: One main contact region after 40 wear steps.

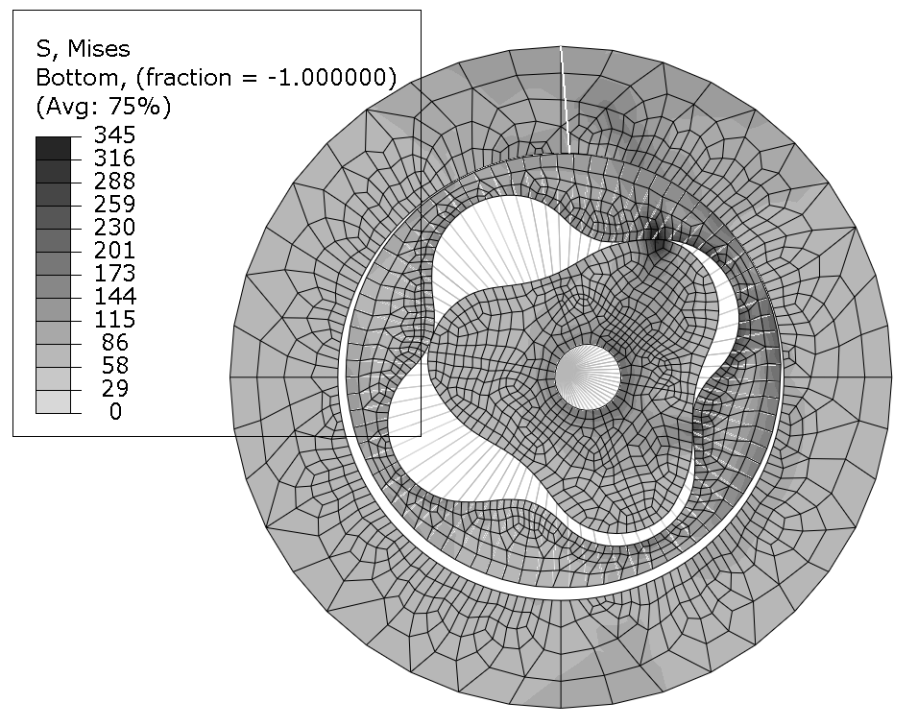

Figure 16: Two main contact regions after 40 wear steps.

With the occurrence of wear, the initial 4 point contact (see in [2]) is immediately reduced to a 2 to 3 point contact situation (see Figures 15 and 16 in which the stress distribution is given only at the contact surfaces). With the transition from a 2 point contact to a 3 point contact, the $\mathrm{x}$-coordinate of the 
center of the outer rotor moves from the minimum to the maximum, as indicated in Figure 14.

With the increasing wear, it can be seen from Figure 14 that the center of the outer rotor gradually approaches to the upper right corner. This can be understood from the motion of the outer rotor by the action of inner rotor. Due to the special configuration of the inner rotor and outer rotor, the main contact regions between the two rotors always occurs at the right half side, which can be seen from with one main contact region and with two main contact regions at the right side. By combining the contact forces and the resulting pressure difference, the outer rotor approaches to the upper right position.

\section{Conclusions}

Based on the wear simulation tool Wear-Processor, the progress of wear in a micro lobe pump has been predicted. The simulation is based on the dynamic behavior of the pump. A single wear step wear simulation as well as a multiple wear step wear simulation was carried out. The results indicate that multiple wear step simulation is essential for correct prediction of wear in such a complex dynamic system. It has been shown that an initial clearance has little influence on the wear distribution on the various surfaces. The predicted gap formation depends nearly linear on the number of rotations.

With increasing wear, the center of the outer rotor moves to the upper right corner. The transition from a 2-point contact to a 3-point contact causes a shift of the $\mathrm{x}$-coordinate of the center of the outer rotor from the minimum to the maximum.

The present work has successfully shown that wear can be predicted in complex dynamic systems. This opens up new possibilities for designers of engineering systems. In particular, it is possible to predict the effect of wear on the changing load, contact conditions, kinematics, and performance of the system under consideration. Furthermore, for given criteria, such as maximum gap size, amount of wear, maximum stress, etc, the simulation results provide a knowledge basis for the estimation of the life time in dependence of the component design and, in this way, can help to avoid expensive trial and error with prototype testing.

\section{Acknowledgement}

The authors would like to thank the German Research Foundation (DFG) for funding this work under transfer project T3 within the scope of the collaborative research center, SFB 499 - Design, production and quality assurance of molded micro-parts constructed from metals and ceramics.

\section{References}

[1] R. Ruprecht, T. Gietzelt, and J. Hausselt, Material and process development for wear loaded micro parts (in German). 2001, Forschungszentrum Karlsruhe: Karlsruhe, Germany. p. 47-54. 
[2] N. Huber and J. Aktaa, Dynamic finite element analysis of a micro lobe pump. Microsystem Technologies, 2003, 9(6-7): p. 465-469.

[3] M. Pfuff and W. Brocks, Simulation of contact and wear in ceramic microsystem parts (in German). 2001, Forschungszentrum Karlsruhe: Karlsruhe, Germany. p. 83-87.

[4] J.A. Williams, Friction and wear of rotating pivots in mems and other small scale devices. Wear, 2001, 251: p. 965-972.

[5] W.G. Sawyer, Surface shape and contact pressure evolution in two component surfaces: Application to copper chemical mechanical polishing. Tribology letters, 2004, 17: p. 139-145.

[6] J. Brauer and S. Andersson, Simulation of wear in gears with flank interference - a mixed FE and analytical approach. Wear, 2003, 254: p. 1216-1232.

[7] K.E. Nürnberg, G. Nürnberg, M. Golle, et al., Simulation of wear on sheet metal forming tools - An energy approach. Wear, 2008, 265(11-12): p. 1801-1807.

[8] V. Hegadekatte, N. Huber, and O. Kraft, Development of a simulation tool for wear in microsystems. Micro-engineering of Metals and Ceramics, Part II: Special Replication Techniques, Automation and Properties, ed. D. Löhe and J. Hausselt. Vol. 4. 2005: Wiley VCH, Weinheim.

[9] V. Hegadekatte, N. Huber, and O. Karft, Finite element based simulation of dry sliding wear. Modelling and Simulation in Materials Science and Engineering, 2005, 13: p. 57-75.

[10] S. Dhanasekaran and R. Gnanamoorthy, Gear tooth wear in sintered spur gears under dry running conditions. Wear, 2008, 265(1-2): p. 81-87.

[11] A. Fllodin and S. Andersson, Simulation of mild wear in helical gears. Wear, 2000, 241(2): p. 123-128.

[12] H.L. Ding and A. Kahraman, Interactions between nonlinear spur gear dynamics and surface wear. Journal of Sound and Vibration, 2007, 307 (3-5): p. 662-679.

[13] V. Hegadekatte, J. Hilgert, O. Kraft, et al., Multi time scale simulation for wear prediction in micro-gears. Wear, 2010, 268(1-2): p. 316-324.

[14] Y.J. Chen and N. Huber, Pressure-force transformation for transient wear simulation in two-dimensional sliding contacts. 2009, submitted.

[15] ABAQUS, Version 6.8.1. 2008, Dassault Systemes Simulia Corp.

[16] V. Hegadekatte, N. Huber, and O. Kraft, Modeling and simulation of wear in a pin on disc tribometer. Tribology letters, 2006, 24(1): p. 51-60. 